PROCEEDINGS OF THE

AMERICAN MATHEMATICAL SOCIETY

Volume 130, Number 6, Pages 1655-1659

S 0002-9939(02)06458-4

Article electronically published on January 28, 2002

\title{
A REMARK ON CALDERÓN-ZYGMUND CLASSES AND SOBOLEV SPACES
}

\author{
DAVID SWANSON
}

(Communicated by David Preiss)

\begin{abstract}
We show how the Sobolev space $W^{k, p}\left(\mathbf{R}^{n}\right)$ may be characterized in terms of the local behavior of its members. We use the local $T^{k, p}$-classes introduced by Calderón and Zygmund.
\end{abstract}

\section{INTRODUCTION}

In their study of the regularity of solutions of elliptic partial differential equations, Calderón and Zygmund [CZ were led to introduce the class $T^{k, p}(x)$. For $x \in \mathbf{R}^{n}, p \geq 1$, and $k \geq-n / p$ (not necessarily an integer), $T^{k, p}(x)$ consists of those functions $f \in L^{p}\left(\mathbf{R}^{n}\right)$ for which there exists a polynomial $P_{x}$ with degree not exceeding $k-1$ such that

$$
\sup _{r>0} r^{-k}\left(f_{B(x, r)}\left|f(y)-P_{x}(y)\right|^{p} d y\right)^{1 / p}<\infty .
$$

$T^{k, p}(x)$ forms a Banach space if, for each $f \in T^{k, p}(x),\|f\|_{T^{k, p}(x)}$ is defined as the sum of $\|f\|_{p}$, the absolute values of the coefficients of the polynomial $P_{x}$ (where $P_{x}$ is written as a Taylor polynomial centered at $x$ ), and the supremum in (1).

For an integer $k \geq 1$, the Sobolev space $W^{k, p}\left(\mathbf{R}^{n}\right), p \geq 1$, consists of all $f \in$ $L^{p}\left(\mathbf{R}^{n}\right)$ whose distributional derivatives $D^{\sigma} f$ of order up to and including $k$ are also members of $L^{p}\left(\mathbf{R}^{n}\right)$. $W^{k, p}\left(\mathbf{R}^{n}\right)$ is a Banach space when normed by

$$
\|f\|_{W^{k, p}\left(\mathbf{R}^{n}\right)}=\sum_{|\sigma| \leq k}\left\|D^{\sigma} f\right\|_{p} .
$$

Calderón and Zygmund showed that for $k \geq 1$ and $1<p<\infty$, a function $f \in W^{k, p}\left(\mathbf{R}^{n}\right)$ belongs to $T^{k, p}(x)$ for almost all $x \in \mathbf{R}^{n}$. It is a natural question to ask what type of converse exists to this statement. Clearly a true converse cannot hold (consider the indicator function of the unit ball in $\mathbf{R}^{n}$ ). On the other hand Bagby and Ziemer [BZ], using distributional arguments due to Calderón [C], showed that if $1<p<\infty$ and $f \in T^{k, p}(x)$ for all $x \in \mathbf{R}^{n}$, then in fact $f \in W^{k, p}\left(\mathbf{R}^{n}\right)$. Their argument further shows that the conclusion remains valid if one assumes

Received by the editors October 2, 2000.

2000 Mathematics Subject Classification. Primary 46E35.

Key words and phrases. Sobolev spaces, Calderón-Zygmund classes.

(C)2002 American Mathematical Society 
only that $f \in T^{k, p}(x)$ for all $x$ outside a compact set with $n-1$ dimensional integralgeometric measure 0 . In the case $k=1$ they used a more sophisticated argument from geometric measure theory to show that the compactness of the exceptional set is not necessary.

The hypotheses of these statements are too strong to be satisfied by typical members of $W^{k, p}\left(\mathbf{R}^{n}\right)$. In this note we determine a relationship between the spaces $W^{k, p}\left(\mathbf{R}^{n}\right)$ and $T^{k, p}(x)$ which in fact characterizes $W^{k, p}\left(\mathbf{R}^{n}\right)$. Instead of considering the size of the exceptional set, we look at bounds on the supremum in (1). We use the $T^{k, p}(x)$ seminorm, defined for $f \in T^{k, p}(x)$ by

$$
|f|_{T^{k, p}(x)}:=\sum_{|\sigma| \leq k-1}\left|D^{\sigma} P_{x}(x)\right|+\sup _{r>0} r^{-k} f_{B(x, r)}\left|f(y)-P_{x}(y)\right| d y .
$$

Hölder's inequality shows that $|f|_{T^{k, p}(x)}<\infty$ whenever $f \in T^{k, p}(x)$, and therefore $|f|_{T^{k, p}(x)}<\infty$ for almost all $x \in \mathbf{R}^{n}$ provided that $f \in W^{k, p}\left(\mathbf{R}^{n}\right)$. We will show that, in this case, $|f|_{T^{k, p}(\cdot)} \in L^{p}\left(\mathbf{R}^{n}\right)$ and that this integrability condition characterizes the relationship between the Calderón-Zygmund classes and the Sobolev spaces.

Theorem 1. Let $k \geq 1$ be an integer and $1<p<\infty$. Then a function $f \in L^{p}\left(\mathbf{R}^{n}\right)$ belongs to $W^{k, p}\left(\mathbf{R}^{n}\right)$ if and only if

1. $f \in T^{k, p}(x)$ for almost all $x \in \mathbf{R}^{n}$; and

2. $|f|_{T^{k, p}(\cdot)} \in L^{p}\left(\mathbf{R}^{n}\right)$.

Moreover, the quantities

$$
\|f\|_{W^{k, p}\left(\mathbf{R}^{n}\right)} \quad \text { and } \quad\left(\int_{\mathbf{R}^{n}}|f|_{T^{k, p}(x)}^{p} d x\right)^{1 / p}
$$

are equivalent.

\section{Proof of Theorem 1}

Let $k \geq 1$ be an integer and let $1<p<\infty$. We first assume that $f \in W^{k, p}\left(\mathbf{R}^{n}\right)$. Calderón and Zygmund's theorem implies that $f \in T^{k, p}(x)$ for almost all $x \in \mathbf{R}^{n}$ and that the polynomial $P_{x}$ is the order $k-1$ distributional Taylor polynomial $T_{x}^{k-1} f$. Applying [BH, Theorem 3] there is a constant $C$ depending only on $n$ and $k$ such that

$$
\left|f(y)-T_{x}^{k-1} f(y)\right| \leq C|x-y|^{k} \sum_{|\sigma|=k}\left(M\left(D^{\sigma} f\right)(x)+M\left(D^{\sigma} f\right)(y)\right)
$$

for almost all $x, y \in \mathbf{R}^{n}$, where $M$ is the usual Hardy-Littlewood maximal operator. Integrating over a ball $B(x, r)$ it follows that

$$
r^{-k} f_{B(x, r)}\left|f(y)-T_{x}^{k-1} f(y)\right| d y \leq C \sum_{|\sigma|=k}\left(M\left(D^{\sigma} f\right)(x)+M\left(M\left(D^{\sigma} f\right)\right)(x)\right)
$$

for almost all $x \in \mathbf{R}^{n}$ and all $r>0$. Therefore

$$
|f|_{T^{k, p}(x)} \leq C\left(\sum_{|\sigma| \leq k-1}\left|D^{\sigma} f(x)\right|+\sum_{|\sigma|=k} M\left(M\left(D^{\sigma} f\right)\right)(x)\right)<\infty
$$


for almost all $x \in \mathbf{R}^{n}$ and, owing to the $L^{p}$ boundedness of the maximal operator,

$$
\left(\int_{\mathbf{R}^{n}}|f|_{T^{k, p}(x)}^{p} d x\right)^{1 / p} \leq C\|f\|_{W^{k, p}\left(\mathbf{R}^{n}\right)} .
$$

Conversely, assume that $f \in T^{k, p}(x)$ for almost all $x \in \mathbf{R}^{n}$ and that

$$
K:=\left(\int_{\mathbf{R}^{n}}|f|_{T^{k, p}(x)}^{p} d x\right)^{1 / p}<\infty .
$$

Let $\varphi \in C_{0}^{\infty}\left(\mathbf{R}^{n}\right)$ be a function supported in the unit ball satsfying $\varphi_{\varepsilon} * P=P$ for every $\varepsilon>0$ and every polynomial $P$ with degree not exceeding $k$, where $\varphi_{\varepsilon}(x)=$ $\varepsilon^{-n} \varphi(x / \varepsilon)$; cf. [Z] Lemma 3.5.6]. Let $f_{\varepsilon}=\varphi_{\varepsilon} * f$ for all $\varepsilon>0$. $f \in L^{p}\left(\mathbf{R}^{n}\right)$ implies that $f_{\varepsilon} \rightarrow f$ in $L^{p}\left(\mathbf{R}^{n}\right)$ as $\varepsilon \rightarrow 0$. Now let $\sigma$ be a multi-index with $|\sigma| \leq k$. Then following Hajłasz [H] we have

$$
\int_{\mathbf{R}^{n}} f \cdot D^{\sigma} \psi d x=\lim _{\varepsilon \rightarrow 0} \int_{\mathbf{R}^{n}} f_{\varepsilon} \cdot D^{\sigma} \psi d x=\lim _{\varepsilon \rightarrow 0}(-1)^{|\sigma|} \int_{\mathbf{R}^{n}} D^{\sigma} f_{\varepsilon} \cdot \psi d x
$$

whenever $\psi \in C_{0}^{\infty}\left(\mathbf{R}^{n}\right)$. If $P$ is a polynomial whose degree does not exceed $k$, then $P * D^{\sigma} \varphi_{\varepsilon}(x)=D^{\sigma} P * \varphi_{\varepsilon}(x)=D^{\sigma} P(x)$ for all $\varepsilon>0$, and therefore

$$
D^{\sigma} f_{\varepsilon}(x)=f * D^{\sigma} \varphi_{\varepsilon}(x)=\left(f-P_{x}\right) * D^{\sigma} \varphi_{\varepsilon}(x)+D^{\sigma} P_{x}(x)
$$

provided $f \in T^{k, p}(x)$. As long as $0<\varepsilon<1$ we have $\varepsilon^{-|\sigma|} \leq \varepsilon^{-k}$, hence

$$
\left|D^{\sigma} f_{\varepsilon}(x)\right| \leq C \varepsilon^{-|\sigma|} f_{B(x, \varepsilon)}\left|f(y)-P_{x}(y)\right| d y+\left|D^{\sigma} P_{x}(x)\right| \leq C|f|_{T^{k, p}(x)}
$$

for all such $x$. This inequality follows from the definition of $|f|_{T^{k, p}(x)}$ when $|\sigma| \leq$ $k-1$, and in the case $|\sigma|=k$ observe that $D^{\sigma} P_{x}(x)=0$ since the degree of $P_{x}$ does not exceed $k-1$. Passing to the limit as $\varepsilon \rightarrow 0$, (2) and Hölder's inequality imply that

$$
\left|\int_{\mathbf{R}^{n}} f \cdot D^{\sigma} \psi d x\right| \leq C K\|\psi\|_{p /(p-1)}
$$

for all $\psi \in C_{0}^{\infty}\left(\mathbf{R}^{n}\right)$, so the Riesz Representation Theorem provides a function $h^{\sigma} \in L^{p}\left(\mathbf{R}^{n}\right)$ with $\left\|h^{\sigma}\right\|_{p} \leq C K$ with the property that

$$
\int_{\mathbf{R}^{n}} f \cdot D^{\sigma} \psi d x=\int_{\mathbf{R}^{n}} h^{\sigma} \cdot \psi d x
$$

for all $\psi \in C_{0}^{\infty}\left(\mathbf{R}^{n}\right)$. Therefore $D^{\sigma} f$ exists and equals $(-1)^{|\sigma|} h^{\sigma}$ for all $|\sigma| \leq k$. It follows that $f \in W^{k, p}\left(\mathbf{R}^{n}\right)$ and that $\|f\|_{W^{k, p}\left(\mathbf{R}^{n}\right)} \leq C K$.

\section{NON-INTEGER SPACES}

It would be interesting to know if an analogue of Theorem 1 can be used as a practical test to determine when functions $f \in L^{p}\left(\mathbf{R}^{n}\right)$ belong to the fractional Sobolev spaces $L^{\alpha, p}\left(\mathbf{R}^{n}\right), \alpha>0[\mathrm{AH}, 1.2 .6]$. That is to say, if $f \in T^{\alpha, p}(x)$ for almost all $x \in \mathbf{R}^{n}$ and $|f|_{T^{\alpha, p}(\cdot)} \in L^{p}\left(\mathbf{R}^{n}\right)$, does it follow that $f \in L^{\alpha, p}\left(\mathbf{R}^{n}\right)$ ? Although the answer to this question is not known, it is possible nevertheless to show that under these hypotheses $f \in L^{\alpha-\varepsilon, p}\left(\mathbf{R}^{n}\right)$ for all $0<\varepsilon<\alpha$. To see this 
we require the following characterization of $L^{\alpha, p}\left(\mathbf{R}^{n}\right)$ due to Strichartz $[\mathrm{S}],[\mathrm{AH}$. 4.8.1]:

Let $1<p<\infty$. If $\alpha>0$ is not an integer, then $f \in L^{\alpha, p}\left(\mathbf{R}^{n}\right)$ if and only if there exist polynomials $P_{x}$ with degree not exceeding $[\alpha]$ such that

$$
S_{\alpha} f(x):=\left(\int_{0}^{\infty}\left(f_{B(x, r)}\left|f(y)-P_{x}(y)\right| d y\right)^{2} \frac{d r}{r^{1+2 \alpha}}\right)^{1 / 2}
$$

is finite for almost all $x \in \mathbf{R}^{n}$ and $\|f\|_{p}+\left\|S_{\alpha} f\right\|_{p}<\infty$.

It is sufficent to prove the result only for small $\varepsilon$, so we choose $0<\varepsilon<\alpha-[\alpha]$. If $f \in T^{\alpha, p}(x)$, then evidently

$$
f_{B(x, r)}\left|f(y)-P_{x}(y)\right| d y \leq r^{\alpha}|f|_{T^{\alpha, p}(x)}
$$

for all $0<r<\infty$. On the other hand, writing $P_{x}$ as a Taylor series centered at $x$ we have

$$
P_{x}(y)=\sum_{|\sigma| \leq[\alpha]} D^{\sigma} P_{x}(x) \frac{(y-x)^{\sigma}}{\sigma !}
$$

which gives the estimate

$$
f_{B(x, r)}\left|f(y)-P_{x}(y)\right| d y \leq r^{[\alpha]}\left(M f(x)+\sum_{|\sigma| \leq[\alpha]}\left|D^{\sigma} P_{x}(x)\right|\right)
$$

provided that $r \geq 1$. Integrating over $(0,1)$ and $(1, \infty)$ separately this shows that $S_{\alpha-\varepsilon} f(x)$ does not exceed the sum of

$$
|f|_{T^{\alpha, p}(x)}\left(\int_{0}^{1} \frac{r^{2 \alpha}}{r^{1+2 \alpha-2 \varepsilon}} d r\right)^{1 / 2}
$$

and

$$
\left(M f(x)+\sum_{|\sigma| \leq[\alpha]}\left|D^{\sigma} P_{x}(x)\right|\right)\left(\int_{1}^{\infty} \frac{r^{2[\alpha]}}{r^{1+2 \alpha-2 \varepsilon}} d r\right)^{1 / 2} .
$$

Since both integrals converge there is a constant $C=C_{\alpha, \varepsilon}$ such that

$$
\begin{aligned}
S_{\alpha-\varepsilon} f(x) & \leq C_{\alpha, \varepsilon}\left(|f|_{T^{\alpha, p}(x)}+M f(x)+\sum_{|\sigma| \leq[\alpha]}\left|D^{\sigma} P_{x}(x)\right|\right) \\
& \leq C_{\alpha, \varepsilon}\left(|f|_{T^{\alpha, p}(x)}+M f(x)\right)
\end{aligned}
$$

Thus if $f \in T^{\alpha, p}(x)$ for almost all $x$ and $f,|f|_{T^{\alpha, p}(\cdot)} \in L^{p}\left(\mathbf{R}^{n}\right)$, then $S_{\alpha-\varepsilon} f \in$ $L^{p}\left(\mathbf{R}^{n}\right)$, hence $f \in L^{\alpha-\varepsilon, p}\left(\mathbf{R}^{n}\right)$. 


\section{REFERENCES}

[AH] Adams, D. R., and Hedberg, L. I., Function Spaces and Potential Theory, Grundlehren der Mathematischen Wissenschaften, Springer, 1996. MR 97j:46024

[BZ] Bagby, T., and Ziemer, W. P., Pointwise differentiability and absolute continuity, Trans. Amer. Math. Soc. 194 (1974), 129-148. MR 49:9129

[BH] Bojarski, B., and Hajłasz, P., Pointwise inequalities for Sobolev functions and some applications, Studia Math. 106 (1) 1993, 77-92. MR 94h:46045

[C] Calderón, A. P., Uniqueness of distributions, Unión Mate. Argentina 25 (1970), 37-55. MR 49:7762

[CZ] Calderón, A. P., and Zygmund, A., Local properties of solutions to elliptic partial differential equations, Studia Math. 20 (1960), 171-225. MR 25:310

[H] Hajłasz, P., Sobolev spaces on an arbitrary metric space, Pot. Anal. 5 (1996), 403-415. MR 97f:46050

[S] Strichartz, R. S., Multipliers on fractional Sobolev spaces, J. Math. Mech. 16 (1967), 10311060. MR 35:5927

[Z] Ziemer, W. P., Weakly Differentiable Functions, GTM 120, Springer-Verlag, 1989. MR 91e:46046

Department of Mathematics, Texas A\&M University, College Station, Texas 77843

E-mail address: dswanson@math.tamu.edu 